\title{
Correlation of Types of Soft Palate, Body Mass Index and Apnoea Hypoapnoea Index in Patients with Sleep Apnoea: A Pilot Study
}

\author{
Dr. Jagdhari. Smriti. B ${ }^{1}$, Dr. Patni V.M. ${ }^{2}$, Dr. Motwani Mukta ${ }^{3}$ \\ (Sr. lecturer ${ }^{1}$, professor ${ }^{2}$, professor and HOD ${ }^{3}$ Dept. Oral Medicine \& Radiology, VSPM'S Dental College \& \\ Research Center, Nagpur. Maharashtra. India.)
}

\begin{abstract}
Aim: Sleep Apnoea a common disorder in young and adults has been studied extensively by pulmonologists, ENT specialists and dentists for various reasons. In the literature it is documented that Sleep Apnoea patient confirmed by polysomography have higher incidence of hook-shaped (S-type) of soft palate.

The aim of our study is to correlate types of soft palate, body mass index (BMI) and apnoea hypoapnoea index (AHI) in sleep apnoea patients.

Materials \& Methods: This preliminary study was carried out in 20 diagnosed patients of sleep apnoea and 20 normal subjects between the age ranges of 12 to 60 yrs in VSPM'S Dental College \& Research Center. The morphology of the soft palate was studied on Digital lateral cephalograms (using Kodak software-72kvp, 12mA, 1 sec exposure). The BMI index, polysomography, Epworth sleepiness scale was recorded in all these patients.

Results: Patients with increase BMI and severe AHI had higher incidence of butt type of palate followed by leaf type. In normal patient the commonest type was found to be leaf type followed by rat tail type.

Conclusion: The present study indicated that severe sleep apnoea patient had higher incidence of butt type of soft palate.

Key Words: Sleep Apnoea, soft palate, lateral cephalogram, body mass index, apnoea hypoapnoea index
\end{abstract}

\section{Introduction}

Obstructive sleep apnoea (OSA) is a potentially life-threatening condition in which periodic cessation of breathing occurs during sleep in the presence of inspiratory effort. This affects not only the quality of life but also has a significant morbidity. ${ }^{1}$

Several cephalometric differences between OSA patients and control samples have been reported. These include mandibular deficiency, bimaxillary retrusion, short cranial base, reduced cranial base angle, mandibular length, increased lower anterior facial height, maxillomandibular plane angle, craniocervical angulation, inferiorly positioned hyoid, and enlarged soft palate. ${ }^{1,2,3,4}$

Pepin et al found the "hooked" appearance of the soft palate in awake patients, which indicated a high risk for obstructive sleep apnoea syndrome (OSAS). In general, most OSA patients seem to have a high body mass index (BMI) shows severe apnoea problems.

\section{Aims \& Objectives}

The aim of our study is to correlate types of soft palate, body mass index (BMI) and apnoea hypoapnoea index (AHI) in sleep apnoea patient.

\section{Materials And Method}

This study comprised of 20 diagnosed patients of sleep apnoea and 20 normal subjects between the age ranges of 12 to 60 yrs in VSPM'S Dental College \& Research Center. The morphology of the soft palate was studied on Digital lateral cephalograms (using Kodak software-72kvp, 12mA, 1sec exposure), and also, Epworth sleepiness scale, BMI index, Polysomnography was recorded.

Patients with congenital anomalies (cleft palate) and having diseases or fracture of the head and neck were excluded from study.

\section{Results}

Present study showed that butt type of soft palate was common in sleep apnoea patient while leaf type was more common in normal population (Table 1/ Fig 1-5) though statistically it is not significant. High BMI index and AHI were commonly seen with butt type of soft palate in apnoea patients. (Table 2)

\section{Discussion}

Lateral cephalometric radiographs have been used by several investigators in an attempt to identify morphological parameters that might be characteristic of OSA. The advantages of Lateral cephalometric 
radiographs are their ease, low cost, normative data available and also they may identify patients with less favorable outcomes after first-line procedures. However, their value as a routine diagnostic tool is uncertain, as a true assessment of the airway dimensions requires a three-dimensional recording technique such as computerized tomography (CT) scanning or magnetic resonance imaging (MRI). ${ }^{1}$ The soft palate is the posterior fibromuscular part of the palate that is attached to the posterior edge of the hard palate. It participates in most oral functions, especially velopharyngeal closure which is related to the normal functions of sucking, swallowing and pronunciation. Body mass index (BMI) provides a simple numeric measure of a person's "fatness" or "thinness" whereas apnoea-hypoapnoea index (AHI) is an index of severity that combines apneas and hypoapnea.

The main symptoms of the obstructive sleep apnoea (OSA) are heavy snoring, excessive daytime somnolence, and disturbed sleep. Several mechanisms have been suggested to explain the relationship between obesity and sleep apnea, including alterations in upper airway structure perhaps caused by fat deposition in the neck, alterations in upper airway function, alteration in the balance between ventilatory drive and load, and obesity-induced hypoxemia. Regardless of the mechanism, it has been well documented that either medical or surgical weight reduction can have a substantial ameliorative impact on OSA. ${ }^{5}$

The risk of OSA rises with increasing body weight, active smoking and age. In addition, patients with diabetes or "borderline" diabetes have up to three times the risk of having OSA. Long-term complications of the sleep apnoea include pulmonary hypertension, cardiac arrhythmias, and heart failure. Some investigators have suggested that cardiac arrhythmias during sleep may be an important cause of death in patients with OSA. In our study, middle aged obese patient had severe AHI. Present study showed that obese patients with OSA show increased upper airway soft tissue dimensions and the results are similar to that, reported by Jean Louis D. Pépin 2

Yu-Fang Liao's ${ }^{4}$ study revealed that in obese group the apnea hypopnea index was significantly related to the soft palate (length $\left[\mathrm{L}_{1}\right]$ and dynamic position change), the hyoid position , the tongue (dynamic position change), and body mass index.

The results of this study are in accordance with Matilde valencia-flores ${ }^{5}$ according to whom sleep apnoea is well known to be associated with obesity and is characterized by repetitive occlusion of the upper airway during sleep. According to J. M. Battagel ${ }^{1}$ the soft palate is longer, larger and in contact with a wider area of the tongue in OSA subjects.

P. Mayer also found, an increased base of the tongue and soft palate width in more obese patients. ${ }^{6}$ Huie Ming Houa revealed the existence of craniofacial and upper airway soft tissue differences in relation to obesity and severity of OSA among male Chinese OSA patients ${ }^{7}$.These finding correlate with our study which suggests that there is correlation between BMI, AHI and butt type of soft palate.

Hooking of the soft palate was defined by pepin et al as an angulation of about $30^{\circ}$ between the distal part of the uvula and the longitudinal axis of the soft palate. They hypothesized that soft palate-hooking plays a key role in pharyngeal collapse, since hooking results in a sudden and major reduction in the oropharyngeal dimensions, which therefore dramatically increases upper airway resistance and the transpharyngeal pressure gradient. Jean Louis D. Pépin ${ }^{2}$ therefore concluded that hooking of the soft palate in awake patients indicates a high risk for OSAS.

Jean Louis D. Pépin ${ }^{2}$ in his study found nine out of 96 patients with OSAS to have hook type of soft palate on awake pharyngeal CT or cephalometric images. In contrast, in present study we found only 1 patient having hook type with severe AHI with obesity whereas butt type of soft palate was found more commonly in sleep apnoea patient. Present study results are similar to that, reported by $\mathrm{M} \mathrm{you}^{3}$ that leaf type of soft palate is more commonly seen in normal population.

\section{Conclusion}

In this study increased BMI and AHI along with butt type of soft palate have been associated with OSA patients. Since this is a pilot study consisting of 20 patients only, statistically significant conclusion cannot be drawn and hence study with larger sample size is required. The variation of the soft palate morphology may be a new explanation for surgical failure and the soft palate may need to be repaired in various patterns. ${ }^{3}$ Dentist and radiologist who observes the finding of hooked/ butt appearance of soft palate on lateral cephalogram should suspect the strong possibility of the presence of OSAS.

\section{References}

[1]. J. M. Battagel and P. R. L'Estrange. The cephalometric morphology of patients with obstructive sleep apnoea (OSA) European Journal of Orthodontics 18 (1996) 557-569

[2]. Jean Louis D. Pépin.Obstructive Sleep Apnea Syndrome: Hooked Appearance of the Soft Palate in Awake Patients-Cephalometric and CT Findings. American Journal of Orthodontics and Dentofacial Orthopedics Volume 91, Issue 5, May 1987, Pages 414-426

[3]. M You, X Li, H Wang Morphological variety of the soft palate in normal individuals: a digital cephalometric study Dentomaxillofacial Radiology (2008) 37, 344-349 
[4]. Yu-Fang Liao ,Ming-Lung Chuang, Chiung-Shing Huang, Ya-Yu Tsai .Upper Airway and Its Surrounding Structures in Obese and Nonobese Patients With Sleep-Disordered Breathing .The Laryngoscope vol 114 issue 6 pages 1052-1059, June 2004

[5]. Matilde Valencia-Flores, Arturo Orea, Violeta A. Prevalence of Sleep Apnea and Electrocardiographic Disturbances in Morbidly Obese Patients .Obesity Research (2000) 8, 262-269

[6]. P. Mayer, J-L. Pépin, G. Bettega, D. Veale, G. Ferretti, C. Deschaux, P. Lévy. Relationship between body mass index, age and upper airway measurements in snorers and sleep apnoea patients. Eur Respir J., 1996, 9, 1801-1809

[7]. Huie Ming Hou, Urban Hagg, Kim Sam, A.B.M. Rabie, Ricky W.K. Wong, B. Lam, Mary S. Ip. Dentofacial Characteristics of Chinese Obstructive Sleep Apnea Patients in Relation to Obesity and Severity. Angle Orthodontist, Vol 76, No 6, 2006

Table 1 - showing comparison between control group and aponea patients

\begin{tabular}{|l|l|cc|cc|}
\hline SN & Pattern & \multicolumn{2}{|c|}{ Control Group } & \multicolumn{2}{|c|}{ Sleep Apnoea } \\
\hline & & \multicolumn{2}{|c|}{ n } & $\%$ & \multicolumn{2}{|c|}{ n } & $\%$ \\
\hline 1 & Butt & 4 & $20 \%$ & 8 & $40 \%$ \\
\hline 2 & Leaf & 11 & $\mathbf{5 5} \%$ & 6 & $30 \%$ \\
\hline 3 & Rat & $\mathbf{5}$ & $\mathbf{2 5} \%$ & 4 & $20 \%$ \\
\hline 4 & S-shape & 0 & $0 \%$ & 1 & $\mathbf{5} \%$ \\
\hline $\mathbf{5}$ & Crook & 0 & $0 \%$ & 1 & $\mathbf{5} \%$ \\
\hline 6 & Straight & 0 & $0 \%$ & 0 & $0 \%$ \\
\hline
\end{tabular}

Chi square value- $0.38, P$ value $-\mathbf{0 . 5 3} p$ value is not significant

Table 2 - showing comparison between image pattern, BMI and AHI score

\begin{tabular}{|c|c|c|c|}
\hline SN & Pattern & BMI & AHI \\
\hline \multirow[t]{4}{*}{1.} & butt - & 5 obese & $2 \bmod$ \\
\hline & & & 3 severe \\
\hline & & 2 over wt & $2 \mathrm{mod}$ \\
\hline & & 1 under wt & 1 mild \\
\hline \multirow[t]{3}{*}{2.} & Leaf - 6 & 2 obese & $2 \mathrm{mod}$ \\
\hline & & 4 over wt & 1 mild \\
\hline & & & $3 \bmod$ \\
\hline \multirow[t]{4}{*}{3.} & Rat tail - 4 & 2 obese & 1 severe \\
\hline & & & 1 mild \\
\hline & & 2 over wt & 1 mild \\
\hline & & & $1 \bmod$ \\
\hline 4. & S-shape- 1 & 1obese & 1 severe \\
\hline 5. & Crook - 1 & 1over wt & $1 \mathrm{mod}$ \\
\hline \multirow[t]{2}{*}{6.} & Straight - 0 & $\mathbf{0}$ & $\mathbf{0}$ \\
\hline & TOTAL - 20 & 20 & 20 \\
\hline
\end{tabular}

Legends

Figure 1: Type 1: "leaf-shaped" (lanceolate); the middle portion of the soft palate is elevated to both the nasoand oro-side

Figure 2: $\quad$ Type 2: "rat-tail shaped"; the anterior portion is inflated and the free margin has an obvious coarctation

Figure 3: Type 3: a "butt-like shape"; the length of the soft palate in this type is about a third to three-quarters of that of the leaf shape. The width has almost no distinct difference from the anterior portion to the free margin Figure 4: Type 5: distorted soft palate, which presents the "S-shape"

Figure 5: Type 6: "crook-shaped" appearance of the soft palate, in which the posterior portion of the soft palate crooks anteriosuperiorly. 


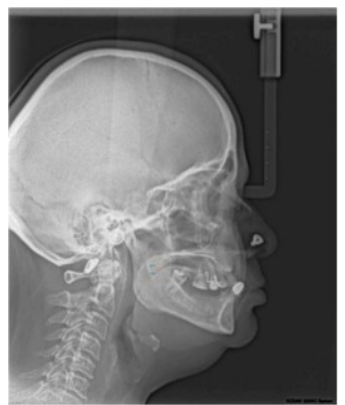

Type1 leaf shape

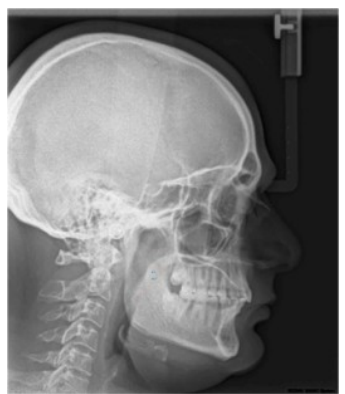

Type 2 rat tail

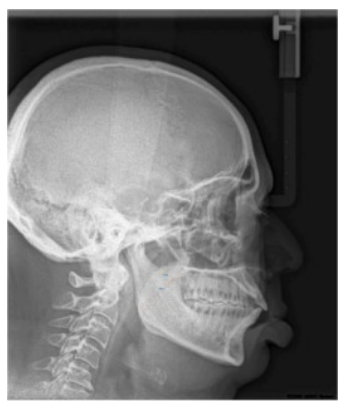

Type 3butt type

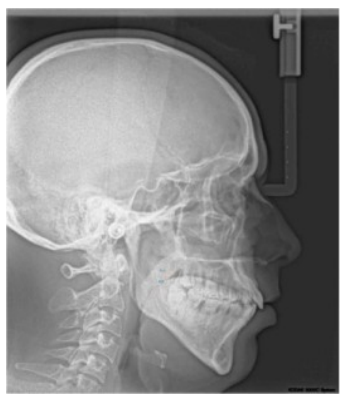

Type 5s shape

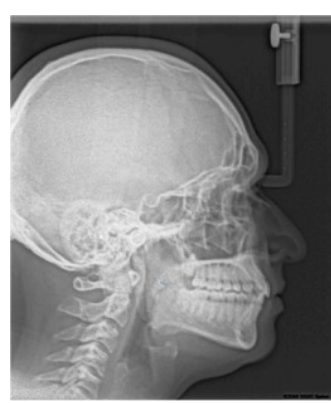

Type 6 crook shape 